\title{
Severe ketorolac-induced asthma diagnosed by chest computed tomography
}

\author{
Vânia Fernandes ${ }^{1}$, Tiago M. Alfaro ${ }^{1}$, João P. Baptista ${ }^{2}$, Frederico S. Regateiro ${ }^{3}$, Fátima Fradinho ${ }^{1}$, Carlos \\ Robalo Cordeiro ${ }^{1}$ \\ ${ }^{1}$ Pneumology Unit A, ${ }^{2}$ Intensive Care Unit, ${ }^{3}$ Immunoallergology Unit, Centro Hospitalar e Universitário de Coimbra, Coimbra, Portugal \\ Correspondence to: Carlos Robalo Cordeiro. CHUC - Pneumologia A, $2^{\circ}$ piso, Praceta Mota Pinto, 300-075 Coimbra, Portugal. Email: carlos.crobalo@gmail.com.
}

\begin{abstract}
Aspirin-exacerbated respiratory disease (AERD) affects $15 \%$ of severe asthmatics and drug reactions cause 200,000 annual deaths in Europe. A 65-year-old lady presented to emergency for progressive abdominal pain. Her medical history included gallstones, asthma, rhinosinusitis and hypertension. She was regularly medicated with inhaled fluticasone, vilanterol and tiotropium, nasal budesonide, pantoprazole, oxazepam and perindopril. She reported partial asthma control and an exacerbation requiring admission to a respiratory ward 6 weeks before. On examination, there was right upper quadrant tenderness and no other changes. Blood tests were normal, and an ultrasound showed gallbladder stones with normal wall. Intravenous ketorolac led to prompt pain resolution. After 30 minutes she became severely dyspnoeic, with an $\mathrm{O}_{2}$ saturation of $85 \%$ on high flow $\mathrm{O}_{2}$. She had no breath sounds on the left lung, and there was no wheezing or prolonged expiration. A chest X-ray showed no pneumothorax and a computed tomography (CT) angiography was performed showing bilateral mucoid impaction and sub-segmental atelectasis. Continuous bronchodilation and systemic steroids led to gradual improving in the following 6 hours. After 9 days of admission on a respiratory ward she was discharged home with no symptoms and normal oxygenation. Importantly, she denied previous allergies to nonsteroidal anti-inflammatory drugs (NSAIDs) and had actually taken diclofenac and nimesulid before with no reactions. This report illustrates both an intravenous NSAID causing severe AERD, and how a chest CT may be instrumental for the diagnosis of life-threatening asthma.
\end{abstract}

Keywords: Asthma; asthma, aspirin-induced; ketorolac; drug hypersensitivity

Submitted Sep 19, 2017. Accepted for publication Oct 20, 2017.

doi: $10.21037 /$ jtd.2017.11.36

View this article at: http://dx.doi.org/10.21037/jtd.2017.11.36

\section{Introduction}

Adverse drug reactions (ADR) remain a concerning cause of morbidity and mortality. Previous studies estimated that about 5\% of all hospital admissions and 200,000 annual deaths are directly caused by ADRs in Europe (1). Aspirin exacerbated respiratory disease (AERD) affects an estimated of $7 \%$ of all adults with asthma (15\% of those with severe forms) and is characterized by asthma, rhinosinusitis with polyposis and respiratory reactions to non-steroid antiinflammatory drugs $(2,3)$. The treatment of this disease consists in nonsteroidal anti-inflammatory drug (NSAID) avoidance and desensitization (4).

\section{Case presentation}

A 65-year-old female presented to the emergency department for progressive abdominal pain, with no other symptoms. Her previous medical history was remarkable for gallstone disease, asthma, rhinosinusitis, hiatal hernia, hypertension, sleep apnoea and venous insufficiency. She was regularly medicated with inhaled fluticasone, vilanterol and tiotropium, nasal budesonide, pantoprazole, oxazepam and perindopril. She used CPAP nightly and reported no previous drug allergies or intolerance. There was a history of previous NSAID intake, namely diclofenac and nimesulid, with no adverse effects. The patient reported 


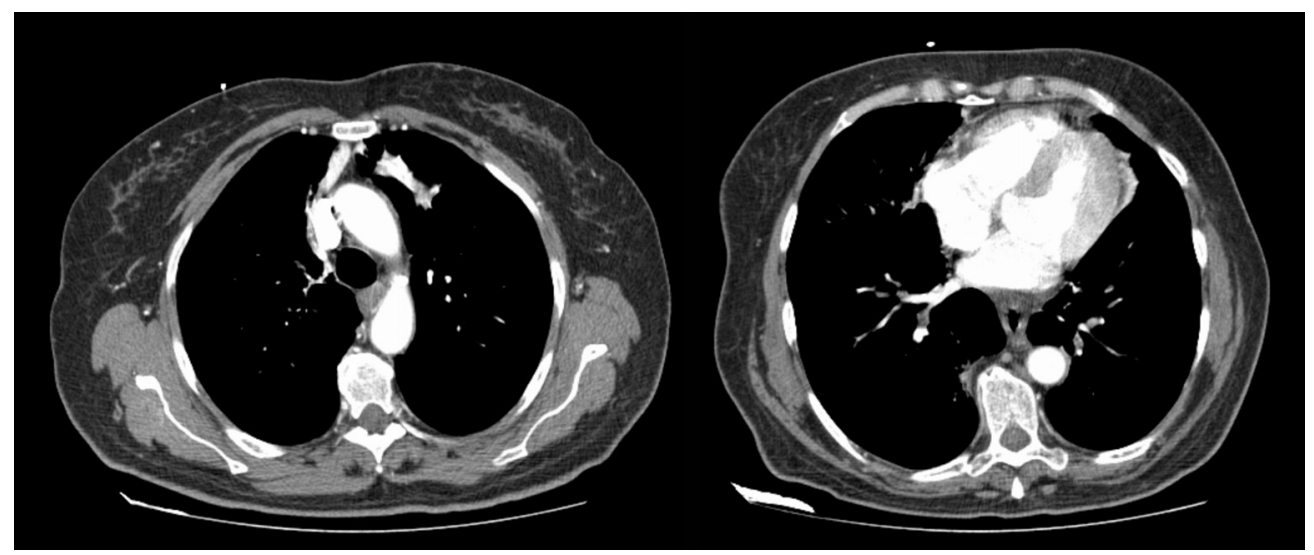

Figure 1 Chest computed tomography showing bilateral upper lobe sub-segmental atelectasis and mucous impaction on lower lobes bronchi.

reasonable control of her asthma, although frequent visits to the emergency department in the last year and an admission to a respiratory ward for an exacerbation 6 weeks before this episode. Her examination revealed normal vital signs and tenderness in the right upper abdominal quadrant with no rebound worsening. Blood tests revealed no changes and an abdominal ultrasound confirmed gallstones with no gallbladder wall changes. A diagnosis of biliary colic was confirmed, and the patient was medicated with intravenous ketorolac, which led to prompt pain resolution. Half an hour after ketorolac administration, the patient complained of progressive dyspnoea and tachycardia. On examination, tachypnoea, tachycardia and absent breath sounds on the left lung field were noted. There was no wheezing or prolonged expiratory time. The peripheral oxygen saturation was $74 \%$ on room air, increasing to $85 \%$ on high flow oxygen (inspired fraction of $60 \%$ ). An emergent chest $\mathrm{X}$-ray showed no signs of pneumothorax or other changes, and an electrocardiograph showed sinus tachycardia. The patient was then put on a non-rebreathing oxygen mask and a computed tomography (CT) angiography was performed. The scan showed diffuse bilateral mucoid impaction of lower lobar bronchi extending to segmental airways. There was also sub-segmental atelectasis in both upper and right middle lobes (Figure 1). No signs of embolism or pneumothorax were noted. The patient remained with $91 \%$ peripheral oxygen saturation and an arterial $\mathrm{PaO}_{2}$ of $53 \mathrm{mmHg}$ on an oxygen inspired fraction of $60 \%$. Upon consultation with Pulmonary Medicine and Intensive Care, it was considered that the most probable diagnosis was ketorolac exacerbated asthma. Intensive therapy was introduced, with inhaled ipratropium bromide, salbutamol and budesonide, and intravenous methylprednisolone, hydroxyzine and aminophylline. During the following 6 hours, the patients progressively improved and was admitted to a respiratory ward. The complaints gradually subsided and the patient was released with no respiratory symptoms and normal oxygen levels after 9 days. She maintains follow-up in Respiratory Medicine and ImmunoAllergology, and is kept under high dose inhaled steroids, bronchodilators and montelukast, with total avoidance of NSAIDs. Due to frequent exacerbations, and poor respiratory function, cross-reactivity to other NSAIDs and aspirin desensitization was not yet performed.

\section{Discussion}

The acute development of dyspnoea and severe hypoxemia in this patient shortly after ketorolac administration is compatible with the diagnosis of ketorolac induced asthma. Importantly, ketorolac was the only drug that was administered before the development of dyspnoea. The patient's chest CT was very helpful in the differential diagnosis of the cause of acute respiratory failure. The presence of lobar collapse and mucoid impaction have been previously described as reversible findings in the CT of asthma patients (5). Furthermore, necropsy descriptions of fatal asthma cases report lung overinflation associated with areas of atelectasis and bronchial and bronchiolar occlusion by mucus plugs (6), both of which were also found in this patient's CT. The CT performed showed rare images of acute alterations in respiratory airways and lung 
parenchyma of asthmatic patients.

This case report also exemplifies how the intravenous administration of a NSAID may lead to serious consequences in asthmatic patients. Ketorolac has been used for pain relief for more than 20 years, due to its opioid like analgesic potency without the typical opioid toxicity, such as respiratory depression and tolerance. Notably, ketorolac displays typical NSAID side-effects including increased bleeding risk and gastro-intestinal and renal effects (7). We found some previous reports of asthma exacerbation after intravenous $(8,9)$, intramuscular (10), and topical (11) administration of ketorolac, including one fatal case (10). These case reports argue for a careful prescription of the drug in patients with asthma, and nasal polyposis, but the broad usage of ketorolac in emergency department may mean that the drug can be used in circumstances where the patient's clinical history is not fully known.

In conclusion, we present the case report of a patient with a life-threatening asthma exacerbation induced by the administration of intravenous ketorolac. This drug should be used with caution in asthmatic patients, especially on those with severe forms and adult onset disease, in whom AERD is more frequent.

\section{Acknowledgements}

None.

\section{Footnote}

Conflicts of Interest: This case report has been previously presented as a poster at the European Respiratory Society International Congress 2017 in Milan, Italy, September 9-13 of 2017 (PA4034).

Informed Consent: Written informed consent was obtained from the subject for this publication.

Cite this article as: Fernandes V, Alfaro TM, Baptista JP, Regateiro FS, Fradinho F, Cordeiro CR. Severe ketorolacinduced asthma diagnosed by chest computed tomography. J Thorac Dis 2017;9(Suppl 16):S1567-S1569. doi: 10.21037/ jtd.2017.11.36

\section{References}

1. Bouvy JC, De Bruin ML, Koopmanschap MA. Epidemiology of adverse drug reactions in Europe: a review of recent observational studies. Drug Saf 2015;38:437-53.

2. Laidlaw TM, Boyce JA. Aspirin-Exacerbated Respiratory Disease--New Prime Suspects. N Engl J Med 2016;374:484-8.

3. Kennedy JL, Stoner AN, Borish L. Aspirin-exacerbated respiratory disease: Prevalence, diagnosis, treatment, and considerations for the future. Am J Rhinol Allergy 2016;30:407-13.

4. Kowalski ML, Asero R, Bavbek S, et al. Classification and practical approach to the diagnosis and management of hypersensitivity to nonsteroidal anti-inflammatory drugs. Allergy 2013;68:1219-32.

5. Paganin F, Trussard V, Seneterre E, et al. Chest radiography and high resolution computed tomography of the lungs in asthma. Am Rev Respir Dis 1992;146:1084-7.

6. Husain AN. The Lung. In: Kumar V, Abbas AK, Aster JC, et al. editors. Robbins and Cotran Pathologic Basis of Disease. Philadelphia, PA: Elsevier Saunders, 2015: 669-726.

7. Vadivelu N, Gowda AM, Urman RD, et al. Ketorolac tromethamine - routes and clinical implications. Pain Pract 2015;15:175-93.

8. Haddow GR, Riley E, Isaacs R, et al. Ketorolac, nasal polyposis, and bronchial asthma: a cause for concern. Anesth Analg 1993;76:420-2.

9. Chen AH, Bennett CR. Ketorolac-induced bronchospasm in an aspirin-intolerant patient. Anesth Prog 1994;41:102-7.

10. Campobasso CP, Procacci R, Caligara M. Fatal adverse reaction to ketorolac tromethamine in asthmatic patient. Am J Forensic Med Pathol 2008;29:358-63.

11. Sitenga GL, Ing EB, Van Dellen RG, et al. Asthma caused by topical application of ketorolac. Ophthalmology 1996;103:890-2. 